\title{
Empirical model for estimating Fourier amplitude spectra of ground acceleration in Taiwan region
}

\author{
Vladimir Sokolov ${ }^{1, *, \dagger}$, Chin-Hsiung Loh ${ }^{2, \ddagger}$ and Kuo-Liang Wen ${ }^{3, \S}$ \\ ${ }^{1}$ National Center for Research on Earthquake Engineering, Taipei, Taiwan \\ ${ }^{2}$ Department of Civil Engineering, National Taiwan University, Taipei, Taiwan \\ ${ }^{3}$ Institute of Geophysics, National Central University, Chung-Li, Taiwan
}

\begin{abstract}
SUMMARY
A collection of ground-motion recordings (1070 acceleration records) of moderate $\left(5.1 \leqslant M_{\mathrm{L}} \leqslant 6.5\right)$ earthquakes obtained during the execution of the Taiwan Strong Motion Instrumentation Program (TSMIP) since 1991 was used to study source scaling model and attenuation relations for a wide range of earthquake magnitudes and distances and to verify the models developed recently for the Taiwan region. The results of the analysis reveal that the acceleration spectra of the most significant part of the records, starting from $\mathrm{S}$-wave arrival, can be modelled accurately using the Brune's $\omega$-squared source model with magnitudedependent stress parameter $\Delta \sigma$, that should be determined using the recently proposed regional relationships between magnitude $\left(M_{\mathrm{L}}\right)$ and seismic moment $\left(M_{0}\right)$ and between $M_{0}$ and $\Delta \sigma$. The anelastic attenuation $Q$ of spectral amplitudes with distance may be described as $Q=225 f^{1.1}$ both for deep (depth more than $35 \mathrm{~km}$ ) and shallow earthquakes. The source scaling and attenuation models allow a satisfactory prediction of the peak ground acceleration for magnitudes $5.1 \leqslant M \leqslant 6.5$ and distances up to about $200 \mathrm{~km}$ in the Taiwan region, and may be useful for seismic hazard assessment. Copyright (C) 2000 John Wiley \& Sons, Ltd.
\end{abstract}

KEY WORDS: acceleration spectra; scaling law, attenuation relation

\section{INTRODUCTION}

The design of buildings and structures in earthquake prone regions must be based on information relating to expected seismic effects expressed in terms of time-domain quantities (maximum amplitudes of ground motion, periods, and duration) and spectral quantities (Fourier spectra and

\footnotetext{
* Correspondence to: Vladimir Sokolov, National Center for Research on Earthquake Engineering, 200 Hsinhai Road, Section 3, Taipei, Taiwan.

${ }^{\dagger}$ Research Associate.

‡ Professor and Director of NCREE.

${ }^{\S}$ Professor.
}

Contract/grant sponsor: National Science Council of the Republic of China; contract/grant number: NSC87-2621-P319-001. 
response spectra). Design of some critical facilities also requires a time function of ground acceleration. Estimation of time domain and spectral parameters of ground motion are obtained either by empirical relations that connect these to earthquake magnitude, distance, and local soil conditions (source scaling and attenuation relations) or by means of mathematical modelling. At present, there is no doubt that these relations are different for different seismic regions. Therefore 'region and site - specific' models should be developed on the basis of available strong ground motion records.

One of the most important parameters describing strong ground motion during earthquakes is the Fourier-amplitude spectrum. Much effort has been devoted recently to the analysis of the frequency content of seismic ground motion in different seismic regions: western and eastern North America [1-6], Caucasian region [7-10] Italy [7, 11], Japan [12], Taiwan [13,14], etc. These assessments were based on data sets that included various number of records and used different models, but they allowed identification of the features of seismic wave excitation and propagation, and, in some cases, estimates to be made of strong ground motion produced by future earthquakes. It is, however, obvious, that source scaling and attenuation models should be tested and updated as new strong ground motion data become available. Therefore, the large amount of ground motion acceleration recordings obtained during the execution of the Taiwan Strong Motion Instrumentation Program (TSMIP) since 1991 [15, 16] provide a unique opportunity to verify the recently developed model [14] and to study source scaling and attenuation relations for a wide range of earthquake magnitudes and distances.

The purpose of this article is parameterization of the spectrum of motion in the frequency band of engineering interest $(0.3-0.4<f<10-15 \mathrm{~Hz})$ by means of simple analytical expressions describing source and propagation for the condition of the so-called 'very hard rock' sites (VHR). Along with empirical records, these VHR source scaling and attenuation models may be used for the estimation of local site response in terms of soil/bedrock spectral ratios (SBSR), estimation of ground motion parameters (peak ground accelerations, response spectra and synthetic acceleration time histories), and seismic hazard assessment.

\section{DATA BASE}

The data set used in this article includes 1070 horizontal components of ground acceleration records obtained during 81 earthquakes $(5.1 \leqslant M \leqslant 6.5)$ which occurred since 1991 at epicentral distances of up to $200 \mathrm{~km}$. The distribution of the accelerograms with respect to magnitude and distance is shown in Figure 1. Detailed site information is not available for most parts of the records, therefore site conditions are not taken into account in this study. Processing of the records consisted of visual inspection of every accelerogram, selection of the significant part of the record starting from S-wave arrival, and the computation of Fourier amplitude spectra using a 10 per cent cosine window. The spectra were smoothed using a three-point running Hanning average filter having a bandwidth of $0.1 \mathrm{~Hz}$ (20 consecutive smoothings were applied to raw spectra). The amplitudes of the spectra were tabulated at frequencies having a spacing of 0.1 log-frequency units, since it is usual to model log amplitude versus log frequency. The records have been obtained by digital accelerographs, and base-line correction procedure was applied to the records. Therefore we assume that long-period noise levels allow us to consider frequencies above $0.2-0.3 \mathrm{~Hz}$. 


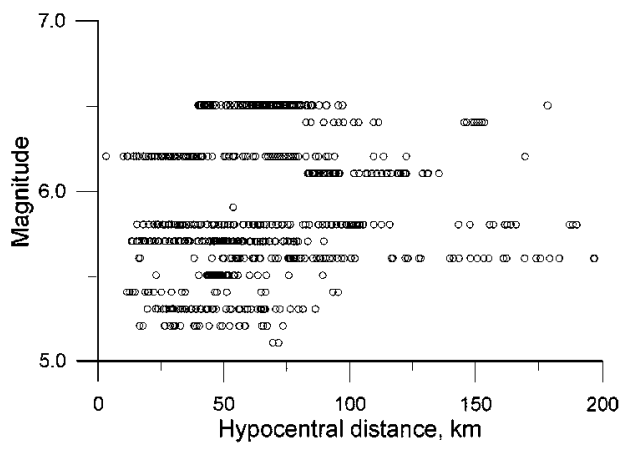

Figure 1. Distribution of the spectral data with respect to the magnitude and distance.

Table I. Number of earthquakes (in parentheses) and records used in this study.

\begin{tabular}{lccc}
\hline & \multicolumn{3}{c}{ Magnitude range } \\
\cline { 2 - 4 } & $5.0<M<5.5$ & $5.5<M<6.0$ & $6.0<M<6.5$ \\
\hline $\begin{array}{l}\text { Shallow events } \\
(\text { depth }<35 \mathrm{~km})\end{array}$ & $201(39)$ & $242(13)$ & $171(7)$ \\
$\begin{array}{l}\text { Deep events } \\
(\text { depth }>35 \mathrm{~km})\end{array}$ & $74(8)$ & $130(12)$ & $218(2)$ \\
\hline
\end{tabular}

The whole data set was divided into three groups by meaning of magnitude, namely: $5.1 \leqslant M \leqslant 5.5,5.6 \leqslant M \leqslant 6.0$, and $6.1 \leqslant M \leqslant 6.5$ and shallow (hypocentral depth less than $35 \mathrm{~km}$ ) and deep (depth more than $35 \mathrm{~km}$ ) events were selected in every group. Table I lists the distribution of the earthquakes and the spectra for these selections.

\section{ANALYSIS}

There are two approaches to describe Fourier acceleration spectra as a function of magnitude, distance and local site condition. The first one is based on statistical analysis of empirical data to estimate an equation of regression $[4,6,7,9,12,17]$. A large number of frequency-dependent regression coefficients of the equation should be determined. It is necessary to possess information about local soil condition for every station, otherwise this regression describes the so-called 'average soil'.

In the second one, the spectrum is represented by simple analytical expressions describing source, propagation, and site effect, separately. Parameters of the expression can be easily determined on the basis of empirical data. This approach has been used in the present study because it allows us to resolve the major goal - developing a general model of acceleration spectra for the condition of 'very hard rock' sites, and it is not necessary to know detailed information on local soil conditions. 
The general model of radiated spectra, describing the Fourier acceleration spectrum $A$ at frequency $f$, can be expressed as follows [18]:

$$
A(f)=(2 \pi f)^{2} C S(f) D(R, f) I(f, M, R)
$$

where $C$ is the scaling factor, $S(f)$ is the source spectrum, $D(R, f)$ is the diminition function and $I(f, M, R)$ represents the frequency-dependent site response which also depends on the intensity of shaking that, in turn, is determined by earthquake magnitude $M$ and distance $R$. The scaling factor is given by

$$
C=\left(\left\langle R_{\theta \phi}\right\rangle F V\right) /\left(4 \pi \rho \beta^{3} R^{b}\right)
$$

where $\left\langle R_{\theta \phi}\right\rangle$ is the radiation coefficient, $F$ is the free surface amplification, $V$ represents the partitions of the vector into horizontal components, $\rho$ and $\beta$ are the density and shear velocity in the source region, and $R$ is the hypocentral distance.

A commonly used source function $S(f)$ in the Brune's model is

$$
S(f)=M_{0} /\left[1+\left(f / f_{0}\right)^{2}\right]
$$

For the Brune's model, the source acceleration spectrum at low frequencies increases as $f^{2}$ and approaches a value determined by $f_{0}$ (corner frequency) and $M_{0}$ at frequencies $f \gg f_{0}$. The value of $f_{0}$ can be found from the relation $f_{0}=4.9 \times 10^{6} \beta\left(\Delta \sigma / M_{0}\right)^{1 / 3}$. Here $\Delta \sigma$ is the stress parameter in bars, $M_{0}$ is the seismic moment in dyne-cm and $\beta$ in $\mathrm{km} / \mathrm{s}$. The level of the spectrum remains approximately constant for frequencies above $f_{0}$ until the cut-off frequency $f_{\max }$ is approached. The amplitude of the spectrum decays rapidly at frequencies above $f_{\max }$.

The function $D(R, f)$ accounts for frequency-dependent attenuation that modifies the spectral shape. It depends on the hypocentral distance $(R)$, regional crustal material properties, the frequency-dependent regional quality factor $Q$ that represents anelastic attenuation, and $f_{\max }$. These effects are represented by the equation

$$
D(R, f)=\exp [-\pi f R / Q(f) \beta] P\left(f, f_{\text {max }}\right)
$$

where $P\left(f, f_{\max }\right)$ is a high-cut filter. Two forms of the $P$ filter are used — the Butterworth filter [18]

$$
P(f)=\left[1+\left(f / f_{\max }\right)^{8}\right]^{-1 / 2}
$$

and that proposed by Anderson and Hough [19]

$$
P(f)=\exp (-\pi \kappa f)
$$

The $f_{\max }$ filter produces more rapid spectral decay than the $\kappa$ (kappa) filter. There are different suggestions regarding the physical meaning of $f_{\max }$ or kappa. Some investigators [20-22] have suggested that it is a source effect, while others $[11,23,24]$ have concluded that kappa is a characteristic quantity associated with the peculiarity of the station site. In all probability, $f_{\max }$ and kappa have both source and site origins because there is evidence for significant regional difference in these quantities [25].

\subsection{Evaluation of quality factor $Q$}

To analyse the dependence between spectral amplitudes, magnitude and stress parameter it is necessary to correct the recorded spectra for the effect of geometrical spreading $1 / R$ and anelastic 
attenuation $Q$ that is written in the form $Q=Q_{0} * f^{n}$. Usually, the so-called 'reference distance' $R=1 \mathrm{~km}$ is used, and the spectra should be multiplied by $R \exp [\pi f R / Q(f) \beta]$ (see Equations (1), (2) and (4)). It has been found recently that the attenuation curve has a bilinear or trilinear shape $[4,6,7]$. When considering geometrical spreading in the form $1 / R^{b}$, attenuation of the direct waves is described using $b=1.0$ for $R_{1}<50-70 \mathrm{~km}$; for the transition zone where a direct wave is joined by postcritical reflections from mid-crustal interfaces and the Moho-discontinuity $\left(50-70<R_{2}<150-170 \mathrm{~km}\right) b=0.0$, and attenuation of multiply reflected and refracted S-waves is described by $b=0.5$ for $R_{3}>170 \mathrm{~km}$. Obviously, the critical distances should be evaluated for every region.

The relations $Q(f)$ for the Taiwan region are given as [26]:

$$
Q(f)=98 f^{1.0} \text { for } H<11 \mathrm{~km}
$$

and

$$
Q(f)=225 f^{1.1} \text { for } H<80 \mathrm{~km}
$$

Tsai [14] used the following relation [27]:

$$
Q(f)=117 f^{0.77}
$$

To choose suitable models for geometrical spreading $\left(1 / R^{b}\right)$ and anelastic attenuation $Q$, the following scheme has been used. Let us consider a data set characterised by approximately the same magnitude $M \pm 0.25$. In this case, besides source-dependent variations (peculiarities of the rupture process), the difference between spectral amplitudes at given frequency is caused by distance-dependent attenuation and site-dependent amplification factors. When partially removing spectral amplitude and shape modification multiplying by $R \exp [\pi f R / Q(f) \beta]$ (i.e. recalculating to 'reference distance'), the scatter of spectral amplitudes, ideally, is determined only by source- and site-dependent factors. Let us suppose that site amplification for the same ground condition does not depend on shaking intensity (i.e earthquake magnitude and distance). Therefore, the proper attenuation model should provide minimum dispersion of the spectral amplitudes $|A|$ at all considered frequencies at 'reference distance'. Thus, it is necessary to analyse values $D_{A}=\left(\Sigma D_{\mathrm{f}}\right) / N_{\mathrm{f}}$ obtained for different attenuation models. Here $D_{\mathrm{f}}$ is the dispersion of $\log _{10}|A|$ values at frequency $f ; D_{\mathrm{A}}$ is the arithmetic average of the $D_{\mathrm{f}}$ values, $N$ is the number of frequencies used. However, the requirement of minimum arithmetic average $D_{\mathrm{A}}$ is not sufficient. Actually, the $D_{\mathrm{A}}$ value does not consider variations of the dispersions at different frequencies, and the optimal attenuation model requires for uniform dispersion of $\log _{10}|A|$ over the whole frequency range. To consider this requirement, it is necessary also to analyse values of the ratio $D_{\mathrm{GA}}=D_{\mathrm{G}} / D_{\mathrm{A}}$, where $D_{\mathrm{G}}=\left(D_{1} \times D_{2} \times \cdots \times D_{N}\right)^{1 / N}$ is the geometric average of the values $D_{\mathrm{f}}$ at frequency $f_{\mathrm{i}}$. The ratio $D_{\mathrm{GA}}$ approaches unity under a condition of approximately equal dispersion values at considered frequencies.

The distributions of $D_{\mathrm{A}}$ and $D_{\mathrm{GA}}$ values for various attenuation models $\left(Q=Q_{0} * f^{n}\right)$ were analysed separately for shallow and deep events. Figure 2 shows the results (distribution of the dispersion characteristics versus $Q_{0}$ and $n$ values) for one of the data subsets ( $5.6 \leqslant M \leqslant 6.0$, deep events). In this magnitude range acceleration records were obtained at hypocentral distances $40-200 \mathrm{~km}$, and depths of earthquake sources vary from $35-80 \mathrm{~km}$. It is seen that variations of distance to transition zone $\left(R_{1}\right)$, where geometric spreading is negligible, produce similar results: values of average dispersion $D_{\mathrm{A}}$ are the least (the minimum value +10 per cent) for $Q_{0}>150-200$ and for a wide range of $n$. Distributions of the dispersion ratio $D_{\mathrm{GA}}$ shows that the 
(a)

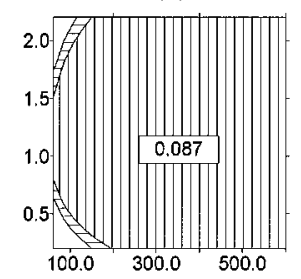

(b)

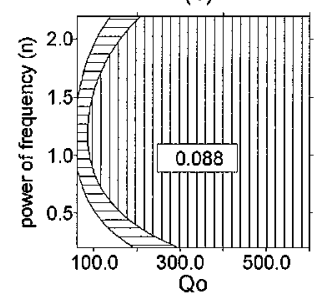

(c)
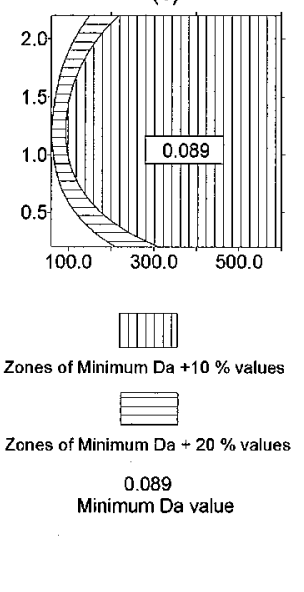

Ratio $\mathrm{Dga}$ of geometric / arithmetic averages (Dg/Da)

(a)

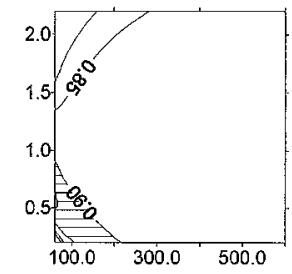

(b)

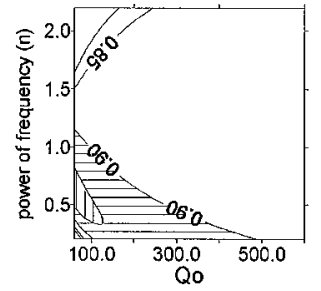

(c)
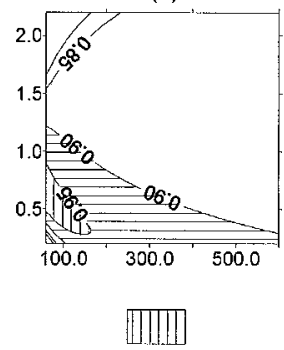

Zone of Dga $>0.95$

Zone of Dga $>0.90$

Zone of Dga $<0.90$

Figure 2. Distribution of the dispersion parameters of the spectral amplitudes at reference distance $R=1 \mathrm{~km}$ for different attenuation models $\left(Q=Q_{0} \times f^{n}\right)$, deep events. (a) distance to transition zone $R_{1}=30 \mathrm{~km}$; (b) $R_{1}=50 \mathrm{~km}$; (c) $R_{1}=70 \mathrm{~km}$.

scatter of spectral amplitudes at different frequencies is homogeneous for small $Q_{0}$ and $n$ values, but the ratio $D_{\mathrm{G}} / D_{\mathrm{A}}$ of more than 0.85 (that may be considered an acceptable value) is also observed for $Q_{0}>100$ and $n<1.8-2.0$. These relations between the characteristics of the dispersion of spectral amplitudes at a reference distance and parameters of attenuation models are slightly different for other magnitudes, but values $Q_{0}>200$ and $n=0.9-1.5$ satisfy the criteria of the optimal attenuation model (minimum $D_{\mathrm{A}}+10$ per cent and $D_{\mathrm{GA}}>0.85-0.9$ ) in all cases for deep events. The solution should be consistent with the typical regional model, and therefore the relation $Q(f)=225 f^{1.1}$ is chosen for deep events in the Taiwan region. The distance to transition zone $R_{1}$ should be at least equal to $50 \mathrm{~km}$ for this $Q$-model.

The attenuation model for shallow earthquakes was chosen assuming characteristics of the dispersion of the whole data set for a given magnitude range. Let us suppose that shallow events spectra at a reference distance should correspond to deep events spectra of the same magnitude. Since the attenuation model for deep events has been established, it was used when determining the model for shallow events. Figure 3 show the results for the data subset of $5.6 \leqslant M \leqslant 6.0$. Parameters of the attenuation models varied only for shallow events (depth $<35 \mathrm{~km}$ ), and the following model was used for deep events: $R_{1}=50 \mathrm{~km}, Q(f)=225 f^{1.1}$. When the whole data set is analysed, the average dispersion $D_{\mathrm{A}}$ is larger than the dispersion obtained when analysing only deep events, and it corresponds to standard deviation of $0.31-0.35 \log$ units. Variations of distance to transition zone $\left(R_{1}\right)$ do not produce distinct differences in the distribution of the dispersion parameter, but $R_{1}>40-50 \mathrm{~km}$ constantly gives better results. Joint consideration of all data sets allows the same attenuation model for deep events to be accepted for shallow events because this model satisfies the criteria of the optimal attenuation model (minimum $D_{\mathrm{A}}+10$ per cent and $\mathrm{D}_{\mathrm{GA}}>0.85-0.9$ ) for all magnitudes. Actually, a lot of other $Q$-models with slightly different parameters $Q_{0}$ and $n$ can be adopted, but the chosen one has an advantage: it coincides with the previously established model [26]. 
Arithmetic average of dispersion (Da)

(a)

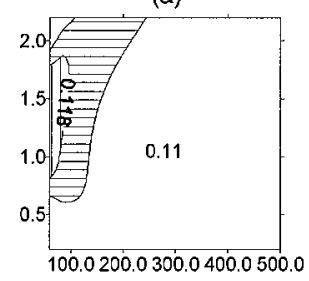

(b)

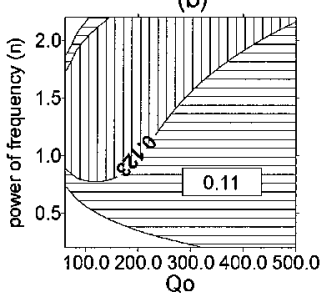

(c)
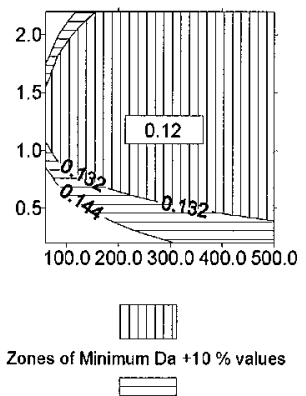

Zones of Minimum $\mathrm{Da}+20 \%$ values

0.11

Minimum Da value
Ratio Dga of geometric / arithmetic averages (Dg/Da)

(a)

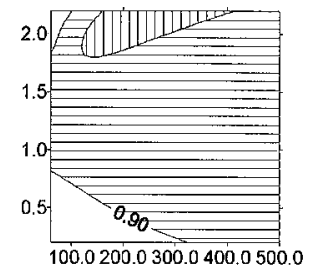

(b)

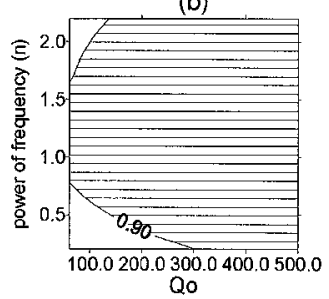

(c)
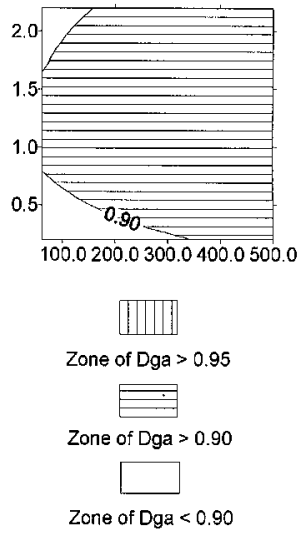

Figure 3. Distribution of the dispersion parameters of the spectral amplitudes at reference distance $R=1 \mathrm{~km}$ for different attenuation models $\left(Q=Q_{0} \times f^{n}\right)$, deep and shallow events. (a) distance to transition zone $R_{1}=30 \mathrm{~km}$; (b) $R_{1}=50 \mathrm{~km}$; (c) $R_{1}=70 \mathrm{~km}$. Attenuation model for deep events: $Q=225 f^{1.1} ; R_{1}=50 \mathrm{~km}$.

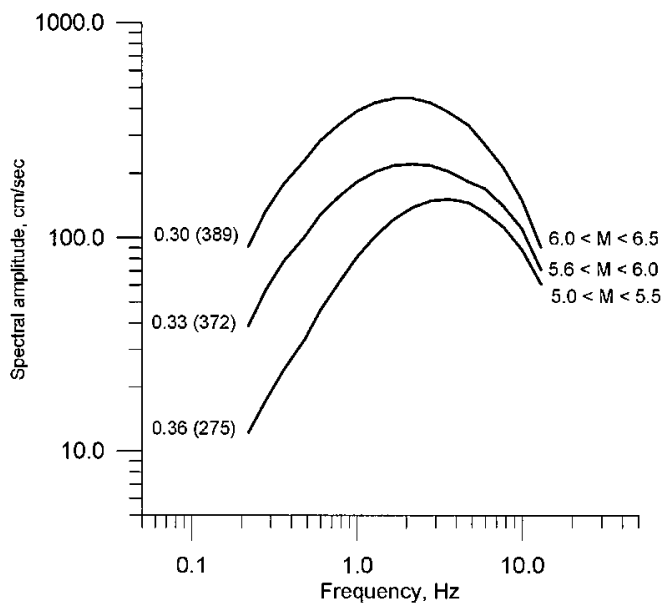

Figure 4. Mean empirical spectra ('average soil') calculated for reference distance $R=1 \mathrm{~km}$. Numbers near the curves denote standard deviation (averaged for the whole frequency range $0.2-13 \mathrm{~Hz}$ ) and number of the spectra used (in parentheses).

The resulting spectra (mean values) at reference distance $R=1 \mathrm{~km}$ for considered magnitude groups are shown in Figure 4. It is seen that in this case the scatter of the data caused by sourceand site-dependent factors, as well as the uncertainties in the distance determination, is relatively low, and the standard deviation does not exceed 0.30-0.36 log unit. As noted before, we do not 
consider soil effects therefore these spectra, averaging the variety of site conditions from rock sites to soft soils of different thickness, correspond to the so-called 'average soil'. These spectra may be used for preliminary prediction of ground motion due to future strong earthquakes at distance $R$ by multiplication by $R^{b} \exp [-\pi f R / Q(f) \beta]$, where $b=-1.0$ for distances $R<50 \mathrm{~km}$, and 0.0 for $50 \leqslant R \leqslant 150-170 \mathrm{~km} ; Q(f)=225 f^{1.1}$.

\subsection{Evaluation of seismic moment-magnitude relation}

Figure 4 shows empirical spectral model for 'average soil'. However, it is necessary to estimate ground motion characteristics for various site conditions. This can be done using a soil/bedrock amplification function, but this approach demands determination of 'bedrock' or 'very hard rock' spectra. In this case (site factor $I(f, M, R)=1$, Equation (1)), the spectral amplitudes at low frequencies are proportional to seismic moment $M_{0}$ and the high-frequency part is controlled by stress parameter $\Delta \sigma$. Thus, it is necessary to determine relations between the magnitude $M_{\mathrm{L}}$, seismic moment and stress parameter. Following Wang [28], there are two relations $M_{0}\left(M_{\mathrm{L}}\right)$ for the Taiwan region

$$
\log _{10} M_{0}=14.571+1.598 M_{\mathrm{L}} \text { after Wang et al. [29] }
$$

and

$$
\log _{10} M_{0}=19.043+0.914 M_{\mathrm{L}} \text { after Li and Chiu [30] }
$$

There is also the well-known Hanks-Kanamori relation [31]:

$$
M_{\mathrm{w}}=\frac{2}{3} \log _{10} M_{0}-10.7
$$

where $M_{\mathrm{w}}$ is moment magnitude. These relations are shown in Figure 5 assuming that moment magnitude $M_{\mathrm{w}}$ is approximately equal to local magnitude $M_{\mathrm{L}}$.

Relations between stress parameter $\Delta \sigma$ and seismic moment $M_{0}$ were determined by Tsai [14] for different seismic zones in the Taiwan region: Northeast ( $\mathrm{N}$ 23.5-26.0, E 121-123), Southeast (21.0-23.5, E 121-123), Northwest (N 23.5-26.0, E 119-121), and Southwest (N 21.0-23.5, E 119-121). Tsai has found that the stress parameter increases in accordance with magnitude (from 65 bar for $M=4.5$ up to 480 bar for $M=6.5$ ), and $\Delta \sigma$ values for the Northeast zone are, in general, smaller than those averaged for the whole Taiwan region. He has also noted that the stress parameters obtained in his study should be treated as upper-boundary values.

Unfortunately, it is not possible to assemble the data set for hard rock sites on the basis of available records. The following assumption was used to obtain a realistic model describing spectra for the condition of a very hard rock (VHR) site. First, every data set used in this study consists of $N$ spectra for earthquakes of magnitudes varying from $M_{\min }$ to $M_{\max }$. Therefore, the spectrum for the central magnitude $M_{\mathrm{C}}=\left(\Sigma M_{\mathrm{i}}\right) / N$, representing ground acceleration for very hard rock, should fit the mean values of empirical 'average soil' spectra at low frequencies $(f<0.1 \mathrm{~Hz})$, and it should generally be less than the mean empirical spectra at higher frequencies. Empirical 'average soil' spectra have been determined for frequencies of more than $0.2 \mathrm{~Hz}$. Therefore, it is necessary to evaluate the amplification (even if roughly) in this frequency range. Let us consider the generalized amplification function for the so-called 'generic soil' (average 


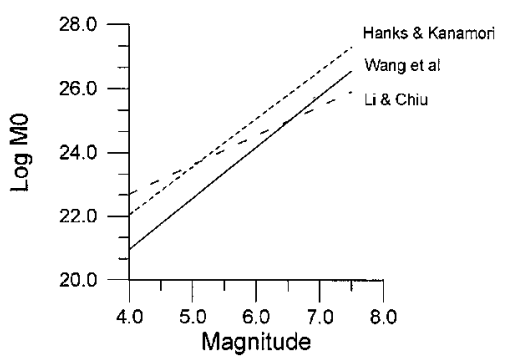

Figure 5. Relations between seismic moment and magnitude for Taiwan region.

shear velocity from the surface to $30-\mathrm{m}$ depth is $310 \mathrm{~m} / \mathrm{sec}$ ) proposed by Boore and Joyner [32]. This model of amplification was developed to be used directly in the stochastic approach, and it is based on shear velocity and density as functions of depth. Although the data used come from restricted geological regions (western and eastern North America), the authors expressed a hope that the result may have a more general applicability. In this case, the amplification is about 1.2-1.3 at $0.1-0.2 \mathrm{~Hz}$. Thus, as general statement for the comparison, 'very hard rock' spectra multiplied by the 'generic soil' amplification function, should fit the mean empirical spectra at low frequencies.

Comparison between empirical spectra and those modelled using various $M_{0}(M)$ relations and stress parameter values at reference distance $R=1 \mathrm{~km}$ is shown in Figures 6 and 7. Modelled spectra were calculated for the central magnitude $\left(M_{\mathrm{C}}\right)$ for the condition of a 'very hard rock' site (density $\rho=2.8 \mathrm{gm} / \mathrm{cm}^{3}$ and shear wave velocity $\beta=3.8 \mathrm{~km} / \mathrm{sec}$ following Hwang and Kanamori [13]). The filter $P(f)=\exp (-\pi \kappa f)$ was used to modify the spectral shape with $k=0.03$ that is usual for rock sites.

When applying the Hanks and Kanamori $M_{0}(M)$ relation and a constant stress parameter $\Delta \sigma \sim 40-50$ bars, the simple Brune's spectral model tends to over-predict the spectra for earthquakes $M>6.0$ at frequencies $f<0.5-1 \mathrm{~Hz}$ (Figure 6). For magnitudes less than 6.0, modelled 'generic soil' spectra fit the mean empirical spectra at low frequencies $(f<1 \mathrm{~Hz})$. This phenomenon is well established for Californian and Eastern US earthquakes [2, 6, 33, 34]. Some models that are more complex than the single-point source (two-corner frequencies, finite-fault simulations) have been developed for these regions to describe the observed spectra. The simple model based on Hanks and Kanamori's relation and combined with the Boore and Joyner amplifications fails to predict the high-frequency part of the spectra. On the one hand, it may be considered as an evidence, that Boore and Joyner's site amplification curves developed for characteristic soil sites in North America cannot be used for the Taiwan region to predict the spectra at intermediate and high frequencies; on the other hand, this demands magnitude and frequency dependence of the parameters of the simple spectral model.

The comparison of empirical spectra and those modelled using the single-frequency Brune model and regional $M_{0} / M$ relation proposed by $\mathrm{Li}$ and Chiu [30] is shown in Figure 7. Stress parameter $\Delta \sigma$ was evaluated using Tsai's relation [14] for the Northeast Taiwan seismic zone

$$
\log _{10} \Delta \sigma=-3.3976+0.2292 \log _{10} M_{0} \pm 0.6177
$$



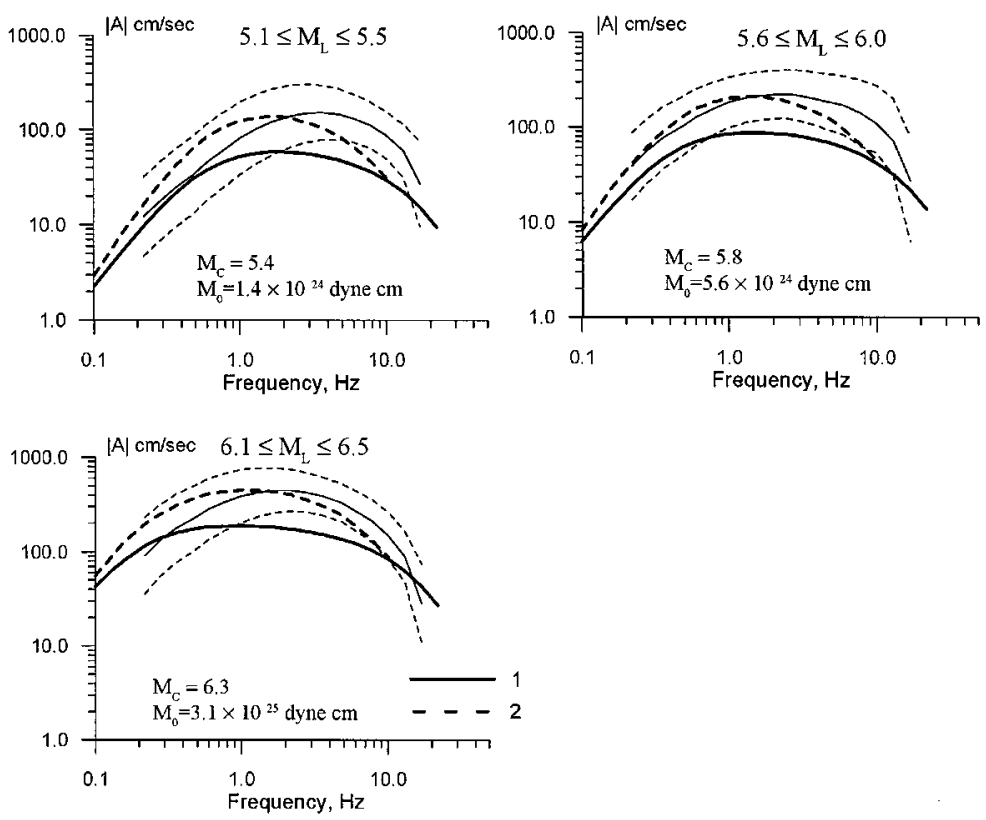

Figure 6. Comparison between empirical spectra and those spectra calculated using $\omega$-squared Brune's spectra model and Hanks \& Kanamori's seismic moment/magnitude relation [31]. Thin lines - empirical spectra: solid line - mean values; dashed lines - mean \pm one standard deviation. Thick lines - modelled spectra: (1) very hard rock site $\left(V_{\mathrm{S}}=3.8 \mathrm{~km} / \mathrm{sec}\right)$; (2) 'generic soil' $\left(V_{\mathrm{S}}=0.31 \mathrm{~km} / \mathrm{sec}\right)$ (after Boore and Joyner [32]). $M_{\mathrm{C}}$ - central magnitude and $M_{0}$ - seismic moment used for calculation of theoretical spectra.

The data sets used in this study include the records of earthquakes which occurred in other seismic zones: for example, in the Southwest Taiwan zone, the stress parameter following Tsai's relations differs sufficiently from that in Northeast Taiwan zone. However, distributions of peak ground accelerations versus magnitude and distance for earthquakes occurring in these zones show the same character. Therefore, bearing in mind that the relation between stress parameter and seismic moment for the Southwest Taiwan seismic zone has been obtained using only a few events, and taking into consideration the relatively large variance of the data predicted by Equation (13) (for example, the stress parameter for earthquake of $M=6.0$ may vary from 30 to 600 bars), the mean value of $\Delta \sigma$ obtained by Equation (13) was used for all data sets. Tsai proposed also regional relations between corner frequency $f_{0}$ and seismic moment, but the values estimated using the relations are close to those obtained by standard equation $f_{0}=4.9 \times 10^{6} \beta$ $\left(\Delta \sigma / M_{0}\right)^{1 / 3}$. When using magnitude-dependent stress parameter, modelled spectra fit the empirical data reasonably well in the low-frequency domain for all magnitudes. Therefore, it is possible to accept these regional relationships between seismic moment and magnitude (Equation (11)) and seismic moment and stress parameter (Equation (13)) for a spectral model in the Taiwan region.

Unfortunately, complete information on the local site condition is not available for every station, but the data set used in this study includes some records from earthquakes of $M=5.6-6.5$ obtained in the so-called 'rock sites' category. Actually, the term 'rock site' implies different 

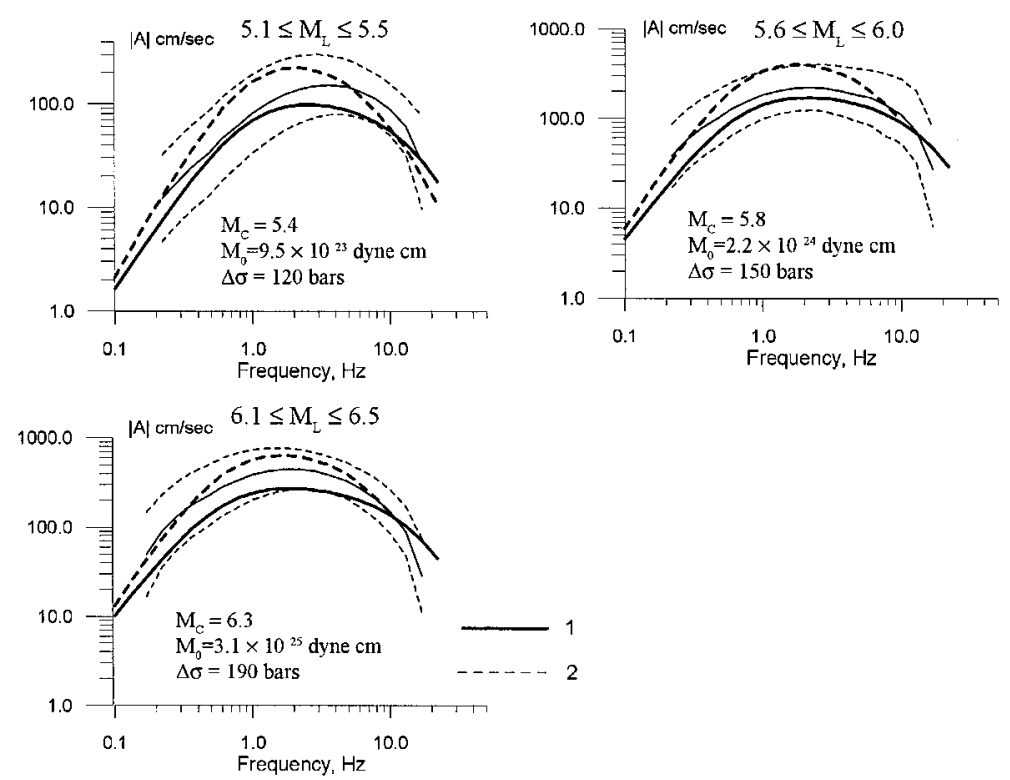

Figure 7. Comparison between empirical spectra and spectra calculated using $\omega$-square Brune's model and Li \& Chiu's seismic moment/magnitude relation [30]. Thin lines — empirical spectra: solid line - mean values; dashed lines - mean \pm one standard deviation. Thick lines — modelled spectra: (1) very hard rock site $\left(V_{\mathrm{S}}=3.8 \mathrm{~km} / \mathrm{sec}\right)$; (2) 'generic soil' $\left(V_{\mathrm{S}}=0.31 \mathrm{~km} / \mathrm{sec}\right)$ (after Boore and Joyner [32]). $M_{\mathrm{C}}$ - central magnitude, $M_{0}$ - seismic moment, and $\Delta \sigma$ - stress parameter used for calculation of theoretical spectra.

geological conditions varying from very stiff rock outcrop to shallow soft layer overlaying weathered and fractured sandstones. Therefore, the records of a single event obtained at different recording sites, even if they are characterized by the same site classification, may reveal some differences due to a site's geological condition, propagation path and source peculiarities. Therefore, instead of comparing the modelled spectrum with a single empirical one, the comparison was made for groups of empirical spectra (including more than 2 records) averaged by means of the site's locations and hypocentral distances. There are three events satisfying this condition, namely: event of 5 June, 1994, $M=6.5, H=5.7 \mathrm{~km}$; event of 25 March 1995, $M=5.6$, $H=76 \mathrm{~km}$, and event of 25 June $1995, M=6.5, H=40 \mathrm{~km}$. We used Li and Chiu's and Tsai's relationships for evaluation of seismic moment and stress parameter for these events. The generalized amplification function for the so-called 'generic rock' (average shear velocity from the surface to 30-m depth is $620 \mathrm{~m} / \mathrm{sec}$ ) proposed by Boore and Joyner [32] are also used to convert 'very hard rock' spectra to 'hard rock' ones.

As can be seen from the comparisons (Figures 8 and 9), when considering the records obtained in far-field zones (source-to-site distance exceeds source dimensions), and when the source-to-site azimuth variation is negligible, both the shape and the amplitude of modelled spectra fit the average observed ones (spectra for the TAP area, events of 25 June 1995 [Figure 8(a)] and 25 March 1995 [Figure 9(a)]; spectra for the TCU area, event of 25 June 1995 [Figure 8(c)]; spectra for the ILA area, event of 5 June 1994 [Figure 9(c)]). Acceleration records in the ILA area for earthquake of 25 June 1995 (Figure 8(b)) were obtained at distances that are comparable to source 

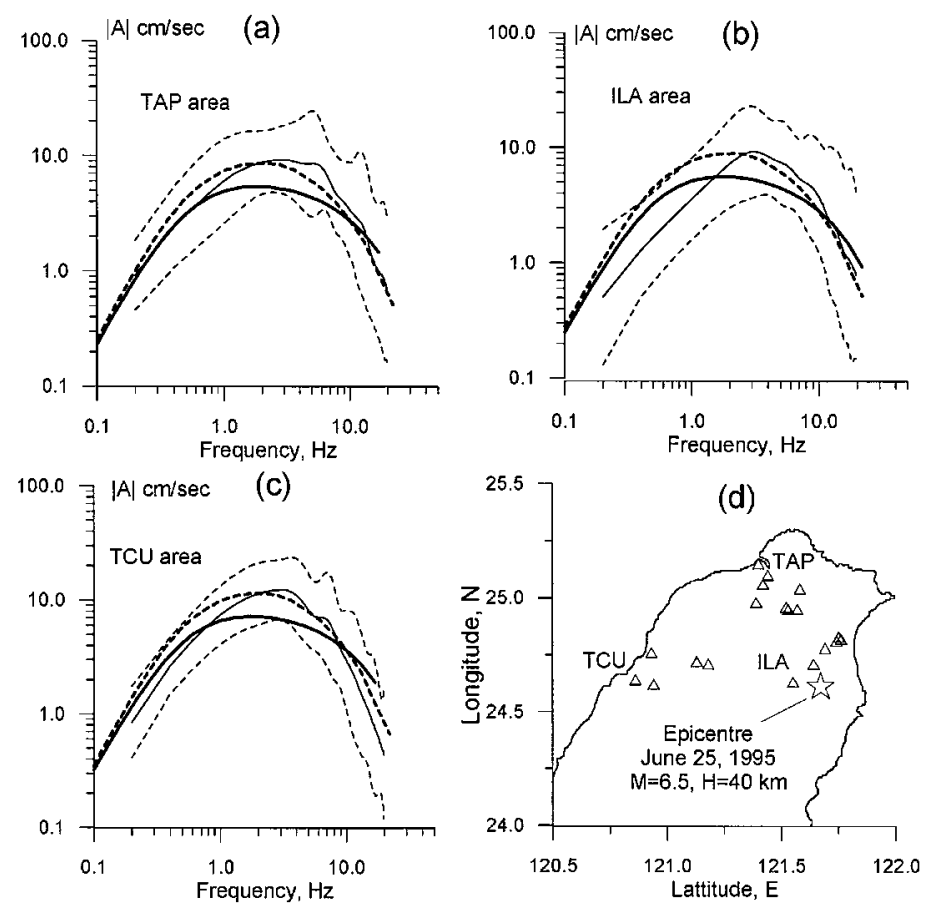

Figure 8. Comparison of theoretical spectra and smoothed observed spectra for stations on rock sites. Event of 25 June $1995, M=6.5, H=40 \mathrm{~km}$, theoretical spectra were modelled using $M_{0}=9.0 \times 10^{24}$ dyne $\mathrm{cm}$ and $\Delta \sigma=200$ bars. Thin lines represent observed spectra: solid line - mean values, dashed lines - mean \pm one standard deviation. Thick lines represent theoretical spectra: solid line — very hard rock site, dashed line - 'generic rock' (after Boore and Joyner [32]). (a) spectra for Taipei area, 16 horizontal components were averaged, the records were obtained at hypocentral distances 55-70 km, reference distance for theoretical spectra $R_{\mathrm{REF}}=65 \mathrm{~km}$. (b) spectra for Ilan area, 12 components were averaged, the records were obtained at hypocentral distances $50-60 \mathrm{~km}, R_{\mathrm{REF}}=55 \mathrm{~km}$. (c) spectra for TCU area, 10 components were averaged, the records were obtained at hypocentral distances $70-80 \mathrm{~km}, R_{\mathrm{REF}}=75 \mathrm{~km}$. (d) map of the station (triangles) and the earthquake epicentre (star).

dimensions (near-field zone). In this case, for frequencies $f<1 \mathrm{~Hz}$, the empirical averaged spectrum has lower spectral amplitudes than that predicted by the Brune's point source model, and theoretical spectra match the empirical one at the high-frequency domain [Figure 8(b)]. The effect of a large finite source, including rupture propagation, directivity and source-receiver geometry may be considered as the reason for the differences.

\section{DISCUSSION}

The obtained source scaling and attenuation models are found, on average, to satisfactorily reproduce the shape and amplitudes of acceleration spectra in the Taiwan region. The suitability of any spectral model and attenuation relation proposed for engineering purposes should be evaluated by the capability of the models to predict peak ground amplitudes (PGA) and response 

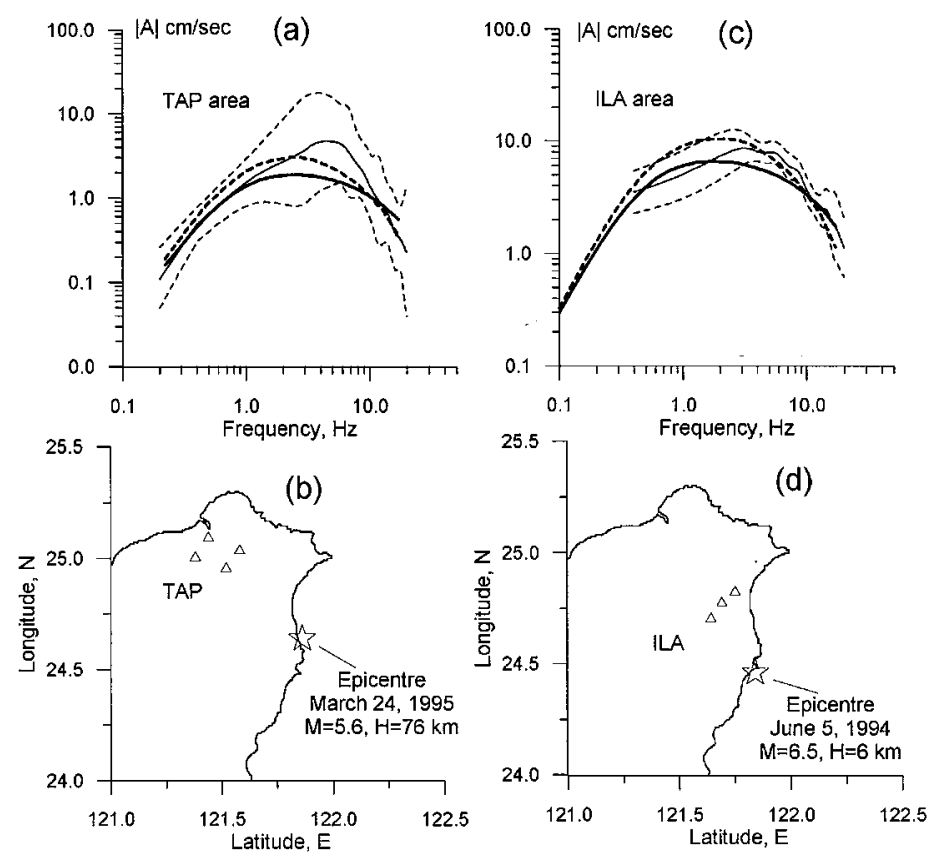

Figure 9. Comparison of theoretical spectra and smoothed observed spectra for some stations on rock sites. Thin lines represent observed spectra: solid line - mean values, dashed lines - mean \pm one standard deviation. Thick lines represent theoretical spectra: solid line — very hard rock site, dashed line — 'generic rock' (after Boore and Joyner [32]). (a-b) event of 24 March 1995, $M=5.6, H=76 \mathrm{~km}$, theoretical spectra were modelled using $M_{0}=1.4 \times 10^{24}$ dyne $\mathrm{cm}$ and $\Delta \sigma=130$ bars. (a) spectra for Taipei area. Eight horizontal components were averaged, the records were obtained at hypocentral distances $90-100 \mathrm{~km}$, reference distance for theoretical spectra $R_{\mathrm{REF}}=100 \mathrm{~km}$. (b) map of the station (triangles) and the earthquake epicentre (star). (c-d) event of 05 June 1994, $M=6.5, H=6 \mathrm{~km}$, theoretical spectra were modelled using $M_{0}=9 \times 10^{24}$ dyne cm and $\Delta \sigma=200$ bars. (c) spectra for Ilan area, 6 components were averaged, the records were obtained at hypocentral distances $30-40 \mathrm{~km}, R_{\mathrm{REF}}=35 \mathrm{~km}$. (d) map of the station (triangles) and the earthquake epicentre (star).

spectra versus earthquake size and distance. In order to test if the spectral models, obtained in this study, are consistent with strong-motion data, the stochastic simulation technique introduced by Boore [18] was used to generate synthetic time histories of ground motion. One of the most important parameters used for stochastic predictions is the duration model because it is assumed that most ( 90 per cent) of the spectral energy given by Equation (1) is spread over a duration $\tau_{0.9}$ of the accelerogram. The frequently used duration models are the following:

$$
\tau_{0.9}=\tau_{0}+b R
$$

where $\tau_{0}$ is the source duration and $b R$ represents a distant-dependent factor to account for dispersion $[35,36]$;

$$
\log _{10} \tau_{0.9}=0.207 M_{\mathrm{S}}+0.264 \log _{10} R-0.65 \pm 0.19
$$


for rock sites, and

$$
\log _{10} \tau_{0.9}=0.178 M_{\mathrm{S}}+0.4 \log _{10} R-0.48 \pm 0.24
$$

for soft soil sites [37]. For eastern North America, Atkinson and Boore [36] used the following parameters: $\tau_{0}=1 /\left(2 f_{0}\right)\left(f_{0}\right.$ is the corner frequency), and $b$ is 0.16 for $10 \leqslant R \leqslant 70 \mathrm{~km}$; -0.03 for $70 \leqslant R \leqslant 130$, and 0.04 for $R \geqslant 130 \mathrm{~km}$. Wen and Yeh [38] studied strong motion duration for the Taiwan region. They obtained the following relationship for ground acceleration

$$
\tau_{0.9}=0.430 \exp \left(0.504 M_{\mathrm{L}}\right) \pm 2.749
$$

for the whole data set including alluvium site and rock site records. Unfortunately, they did not present the relationships between the duration and distance, although they did mention that the duration has a slight tendency to increase with distance up to about $80 \mathrm{~km}$.

Comparisons of individual observed peak amplitudes (both horizontal components) and predicted PGA for different site conditions ('very hard rock' and 'average soil') are shown in Figures 10 and 11. Simulated PGA values were obtained using various duration models and were averaged from 40 simulations. When calculating PGAs for very hard rock sites, the spectral

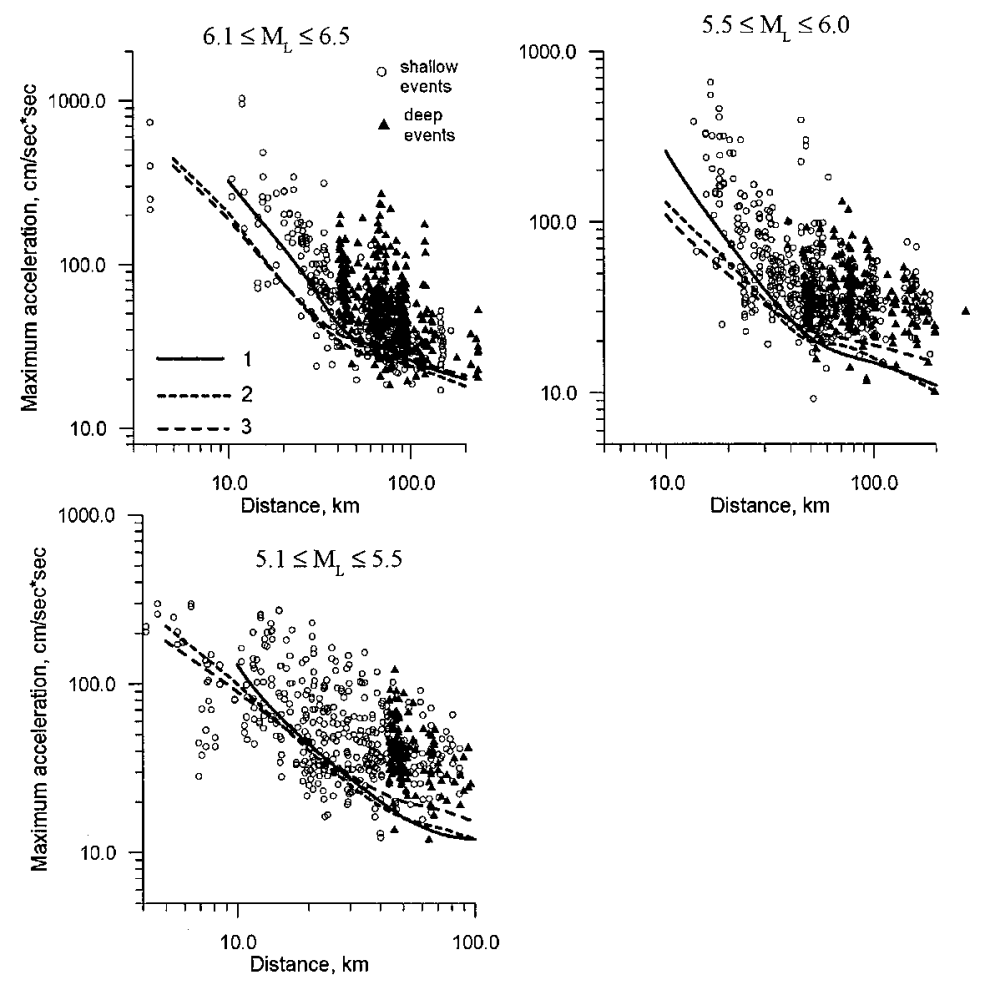

Figure 10. Comparison of observed (symbols) and simulated for condition of very hard rock (lines) peak ground accelerations. Duration models: (1) Atkinson and Boore [36]; (2) Schteinberg [37]; (3) Wen and Yeh [38]. 
model for reference events of central magnitude (see Figure 7) was used. Estimations of peak amplitudes for 'average soil' sites were obtained using mean empirical spectra for reference distance $R=1 \mathrm{~km}$ (Figure 4) recalculated to required distance $R$ using expression $R^{b} \exp [-\pi f R / Q(f) \beta]$. For attenuation model the following parameters were used: $b=-1.0$ for distances $R<50 \mathrm{~km}$, and 0.0 for $50 \leqslant R \leqslant 150-170 \mathrm{~km} ; Q(f)=225 f^{1.1}$.

Actually, distribution of recorded PGA values shown in Figures 10 and 11 reflects the influence of various site conditions. As a general statement for comparisons, peak acceleration for hard rock conditions should be commonly less than those registered at soil sites. Figure 10 shows that PGA values, predicted for hypothetical 'very hard rock' using established source scaling and attenuation models, satisfy this condition. PGA values predicted for conditions of 'average soil' lay almost in the centre of the observed data, and those, calculated using mean + one standard deviation spectral amplitudes exceed generally empirical PGAs (Figure 11). A measure of how well the 'average soil' spectral model fits the empirical data is provided in Figure 12 which plots the residuals (defined as the difference, in log units, between the model predictions and observation) as a function of hypocentral distance. The residuals are shown for the duration models provided by Atkinson and Boore [36] and Wen and Yeh [38]. Table II lists standard deviation of

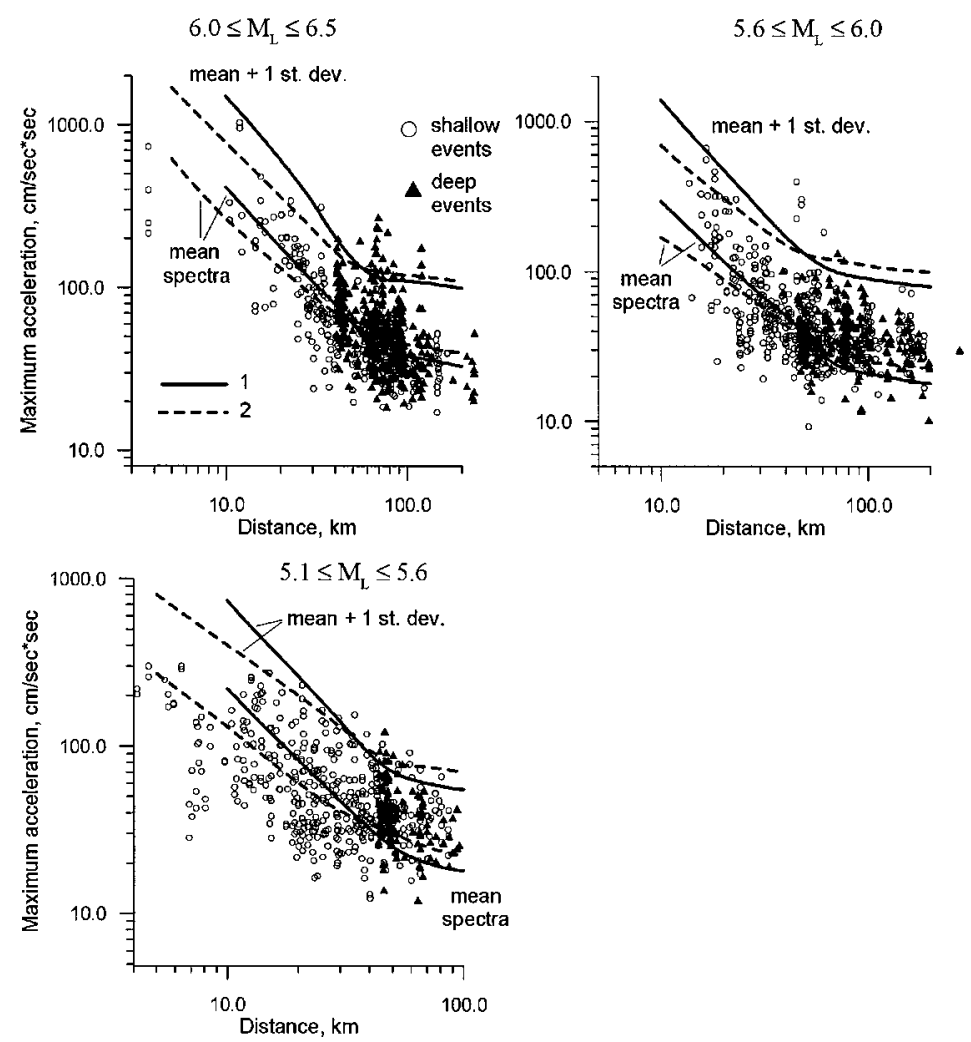

Figure 11. Comparison of observed (symbols) and simulated for 'average soil' condition (lines) peak ground accelerations. Duration models: (1) Atkinson and Boore [36]; (2) Wen and Yeh [38]. 

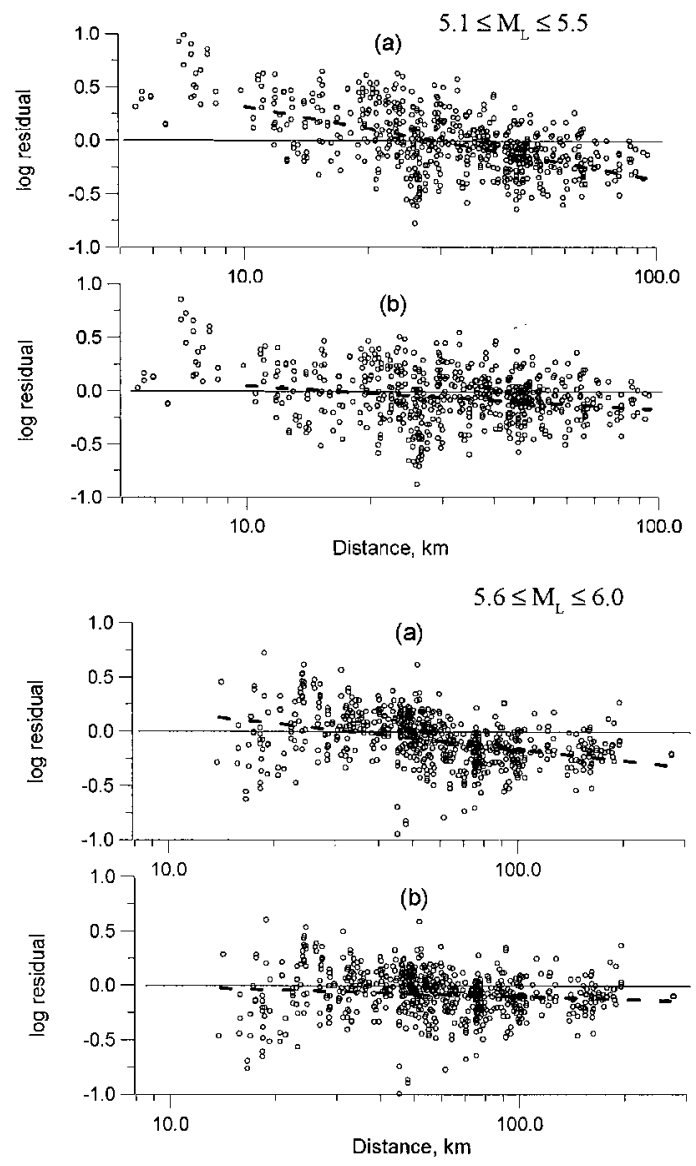

Figure 12. Residuals for peak ground acceleration as a function of hypocentral distance presented for two data subsets. The residuals are defined as the ratio between model predictions and observations. Duration models: (a) Atkinson and Boore [36]; (b) Wen and Yeh [38]. Dashed thick lines show general trend of the residuals.

Table II. Standard deviations (log units) of residuals between modeled and recorded peak ground accelerations.

\begin{tabular}{lccc}
\hline & \multicolumn{3}{c}{ Magnitude range } \\
\cline { 2 - 4 } & $5.0<M<5.5$ & $5.5<M<6.0$ & $6.0<M<6.5$ \\
\hline $\begin{array}{l}\text { Atkinson and Boore } \\
\text { duration model }\end{array}$ & 0.34 & 0.24 & 0.21 \\
$\begin{array}{l}\text { Wen and Yeh } \\
\text { duration model }\end{array}$ & 0.25 & 0.22 & 0.21 \\
\hline
\end{tabular}


the residuals. Atkinson and Boore's [36] duration model tends to overestimate peak amplitudes at small distances and to underestimate those at large distances for earthquakes of $M \leqslant 6.0$, while Wen and Yeh's regional model works well for the whole range of considered distances and shows the smallest standard deviations of the residuals that do not exceed $0.3 \log$ units. It should be noted, that the PGA attenuation model for the Taiwan area obtained recently by Loh and Jean [39] is characterized by a standard deviation of $0.54 \log$ unit.

\section{CONCLUSION}

In this study a collection of ground-motion recordings (1070 acceleration records) of moderate $\left(5.1 \leqslant M_{\mathrm{L}} \leqslant 6.5\right)$ earthquakes obtained during the execution of the Taiwan Strong Motion Instrumentation Program (TSMIP) since 1991 was used to evaluate a source scaling model and attenuation relations for the Taiwan region. The proposed empirical models for estimating Fourier amplitude spectra in the Taiwan region allow satisfactory prediction of the peak ground acceleration for magnitudes $5.1 \leqslant M_{\mathrm{L}} \leqslant 6.5$ and distances up to about $200 \mathrm{~km}$. Source scaling models have been developed for two site conditions: 'very hard rock' (VHR) and 'average soil', but it is possible to use the available soil amplification curves to combine with 'very hard rock' spectra. From this point of view, the proposed VHR model seems to be useful in estimating the soil amplification function in terms of soil/bedrock spectral ratios (SBSR) both for a variety of stations on the same soil category (generalized amplification curve) and for any individual site [40]. These empirical SBSR function in combination with 'very hard rock' spectra can provide a basis for 'regional site-dependent' estimation of seismic hazard (probabilistic and deterministic) in terms of ground-motion parameters used for engineering decisions (peak ground acceleration, response spectra and synthetic accelerograms). These are the topics of future research.

The principle result derived from the study of the features of strong-ground-motion excitation and propagation is the evidence for the necessity for spectral zonation. At the same time, a simple model based on the $\omega$-squared source spectra with region-dependent parameters, despite its simplicity, is an efficient tool for describing the general features of earthquake ground motion.

\section{ACKNOWLEDGEMENTS}

The authors are very grateful to anonymous reviewers for their comments and suggestions for improving the manuscript. This study was carried out at the National Center for Research on Earthquake Engineering and was supported by the National Science Council of the Republic of China under grant NSC87-2621-P-319001.

\section{REFERENCES}

1. Boore DM. Short-period P- and S-wave radiation from large earthquakes: implicatons for source scaling relations. Bulletin of the Seismological Society of America 1986; 76(1):43-64.

2. Boatwright J, Choy G. Acceleration source spectra anticipated for large earthquakes in northeastern North America. Bulletin of the Seismological Society of America 1992; 82(2):660-680.

3. Boore DM, Joyner W, Wennerberg L. Fitting the stochastic omega-squared source model to observed response spectra in western North America: trade-offs between stress drop and kappa. Bulletin of the Seismological Society of America 1992; 82: 1956-1963. 
4. Atkinson GM, Mereu RF. The shape of ground motion attenuation curves in southeastern Canada. Bulletin of the Seismological Society of America 1992; 82(5):2014-2031.

5. Atkinson GM. The high-frequency shape of the source spectrum for earthquakes in eastern and western Canada. Bulletin of the Seismological Society of America 1996; 86(1a):106-112.

6. Atkinson GM, Silva W. An empirical study of earthquake source spectra for California earthquakes. Bulletin of the Seismological Society of America, 1997; 87(1):97-113.

7. Chernov Yu K. Strong Ground Motion and Quantitative Assessment of Seismic Hazard. Fan Publishing House: Tashkent, 1988 (in Russian).

8. Sokolov V Yu. Empirical model for estimating Fourier-amplitude spectra of ground acceleration in the Northern Caucasus (Racha seismogenic zone). Bulletin of the Seismological Society of America 1997; 87(6):1401-1412.

9. Sokolov Y Yu. Ground acceleration spectra from Caucasus earthquakes. Izvestiya, Physics of the Solid Earth 1998; 34(8):663-675.

10. Sokolov V Yu. Spectral parameters of the ground motions in Caucasian seismogenic zones. Bulletin of the Seismological Society of America 1998; 88(6):1437-1444.

11. Rovelli A, Bonamassa O, Cocco M, Di Bona M, Mazza S. Scaling laws and spectral parameters of the ground motion in active extensional areas in Italy. Bulletin of the Seismological Society of America 1998; 78(2):530-560.

12. Kamiyama M. Regression analyses of strong-motion spectra in terms of a simplified faulting source model. In Engineering Seismology and Site Response, Cakmak AS, Herrera I. (eds). Southampton, Boston 1989; 113-126.

13. Hwang LJ, Kanamori H. Teleseismic and strong-motion source spectra from two earthquakes in eastern Taiwan. Bulletin of the Seismological Society of America 1989; 79:935-944.

14. Tsai CCP. Relationships of seismic source scaling in the Taiwan region. Terrestrial and Atmospheric Ocean 1997; 8(1):49-68.

15. Shin TC. Progress summary of the Taiwan strong motion instrumentation program. Symposium on Taiwan Strong Motion Instrimentation Program 1993; 1-10.

16. Kuo KW, Shin TC, Wen KL. Taiwan strong motion instrumentation program (TSMIP) and preliminary analysis of site effect in Taipei basin from strong motion data. In Urban Disaster Mitigation: the Role of Engineering and Technology, Cheng FY, Sheu MS, (eds). Elsevier:Amsterdam, 1995;47-62.

17. Trifunac MD, Lee VW. Empirical models for scaling Fourier amplitude spectra of strong ground acceleration in terms of earthquake magnitude, source to station distance, site intensity and recording site conditions. International Journal of Soil Dynamics and Earthquake Engineering 1989; 8:110-125.

18. Boore DM. Stochastic simulation of high frequency ground motion based on seismological model of the radiated spectra. Bulletin of the Seismological Society of America 1983; 73:1865-1894.

19. Anderson J, Hough S. A model for the shape of the Fourier amplitude spectrum of acceleration at high frequencies. Bulletin of the Seismological Society of America 1984; 74:1969-1993.

20. Papageourgiou AS, Aki K. A specific barrier model for the quantitative description of inhomogeneous faulting and the prediction of strong ground motion. Part 1: description of the model. Bulletin of the Seismological Society of America 1983; 73:693-722.

21. Campillo M. Numerical evaluation of near-field, high-frequency radiation from quasi-dynamic circular faults. Bulletin of the Seismological Society of America 1983; 73(3):723-734.

22. Papageougiou AS. On two characteristic frequencies of acceleration spectra: patch corner frequency and $f_{\max }$. Bulletin of the Seismological Society of America 1988; 78(2):509-529.

23. Hanks T. $f_{\max }$. Bulletin of the Seismological Society of America, 1982; 72:1867-1879.

24. Frankel A. Simulating strong motions of large earthquakes using recordings of small earthquakes: the Loma Prieta mainshock as a test case. Bulletin of the Seismological Society of America 1995; 85(4):1144-1160.

25. Boore DM, Atkinson GM. Stochastic prediction of ground motion and spectral response parameters at hard rock sites in eastern North America. Bulletin of the Seismological Society of America 1987; 77(2):440-467.

26. Wang JH. Q values of Taiwan: a review. Journal of the Geological Society of China, 1993; 36(1):15-24.

27. Chen KC, Chin TC, Wang JH. Estimates of coda Q in Taiwan. Proceedings of the Geological Society of China 1989; 32:339-353.

28. Wang JH. Magnitude scales and their relations for Taiwan earthquakes: a review. Terrestrial and Atmospheric Ocean 1992; 3(4):449-468.

29. Wang JH, Liu CC, Tsai YB. Local magnitude determined from a simulated Wood-Anderson seismograph. Tectonophysics 1989; 166:15-26.

30. Li C, Chiu HC. A simple method to estimate the seismic moment from seismograms. Proceedings of the Geological Society of China 1989; 32:197-207.

31. Hanks TC, Kanamori H. A moment magnitude scale. Journal of Geophysical Research 1979; 84:2348-2350.

32. Boore DM, Joyner WB. Site amplifications for generic rock sites. Bulletin of the Seismological Society of America 1997; 87(2):327-341.

33. Atkinson GM. Earthquake source spectra in Eastern North America. Bulletin of the Seismological Society of America 1993; 83(6):1778-1798. 
34. Schneider J, Silva W, Stark C. Ground motion model for 1989 M 5.9 Loma Prieta earthquake including effect of source, path and site. Earthquake Spectra 1993; 9:251-287.

35. Herrmann RB. An extension of random vibration theory estimates of strong ground motion to large distances. Bulletin of the Seismological Society of America 1985; 75:1447-1453.

36. Atkinson GB, Boore DM. Ground motion relations for eastern North America. Bulletin of the Seismological Society of America 1995; 85(1):17-30.

37. Shteinberg VV. Ground motion parameters during strong earthquakes. Detailed Engineering-Seismological Research (Engineering Seismology Problems, iss. 27). Nauka Publishing House: Moscow, 1986; 7-22 (in Russian).

38. Wen KL, Yeh YT. Characteristics of strong motion durations in the SMART1 array area. Terrestrial and Atmospheric Ocean 1991; 2(3):187-201.

39. Loh CH, Jean W Yu. Seismic zoning on ground motions in Taiwan area. Proceedings of 4th International Conference on Soil Mechanics and Foundation 1997; 71-79.

40. Sokolov V Yu. Rough estimation of site response using earthquake ground motion records. Proceedings of the Second International Symposium on the Effects of Surface Geology on Seismic Motion. Yokohama, Japan, 1-3 December 1998; 517-522. 